\title{
Antenna systems for small spacecraft radio data communication channels
}

\author{
Alexander Generalov ${ }^{1,}{ }^{*}$,Elchin Gadzhiev ${ }^{1}$, Elena Ovchinnikova ${ }^{2}$, Nguen Dinh $\mathrm{To}^{2}$, \\ Svetlana Kondratieva ${ }^{2}$, and Pavel Shmachilin ${ }^{2}$ \\ ${ }^{1}$ JSC "Research Institute for Electromechanics",143502, st. Panfilov 11, Russia \\ ${ }^{2}$ Moscow Aviation Institute (National Research University) "MAI", 125993 \\ Volokolamskoe Highway, 4, Russia
}

\begin{abstract}
Antenna-feeder devices are used on spacecraft, which are part of various on-board systems and complexes. One such system is a radio communication channel for transmitting target information. A model of the aerial array of the radio data communication of target information from waveguide transmitters is developed and its characteristics are determined.
\end{abstract}

\section{Development potential of antenna systems for small spacecraft radio data communication channels}

One of the main tasks is to ensure data exchange between the spacecraft (SC) and ground command facilities. The quality and continuity of communication sessions between the spacecraft and ground facilities depends on the fulfillment of the assigned task on the spacecraft [1].

As a rule, transmit/receive antennas are used onboard the spacecraft and acre equipped with the following systems [2]:

- telemetery system;

- telecommand system;

- navigation systems (GLONASS, GPS);

- radio data communication of target information;

- intersatellite communications;

- $\quad$ special systems (for example, COSPAS-SARSAT);

- scientific systems and complexes, etc.

This research is devoted to the state and development potential of antenna-feeder devices of small spacecraft radio data communication of target information.

With the growing volume of tasks to be solved with the help of space technologies, the requirements for small spacecraft radio data communication of target information changed. This change consists in a sharp increase in the required data transfer speed from the spacecraft to ground data receiving terminal.

In turn, certain restrictions are caused by the rather short time of the communication session of the spacecraft with the data receiving terminal (about 10-15 minutes), as well as a limited number of data receiving terminals [3].

* Corresponding author: oea8888@gmail.com 
Thus, there is an urgent and crucial task to develop high-speed and ultra-high-speed data communication channels.

The work considers the development of the radio data communication of target information antenna system as in the case with the small spacecraft "Kanopus-V" No. 1 [45].

When developing the antenna-feeder device for the radio data communication of target information built based on the Kanopus- $\mathrm{V}$ platform, two transmitters and two centimeterwave reflector antennas were used.

Thus, this approach allows reaching $61.44 \mathrm{Mbit} / \mathrm{s}$ and $122.88 \mathrm{Mbit} / \mathrm{s}$ of data communication speed.

However, the currently used approach to the construction of antenna-feeder device for the radio data communication of target information has several disadvantages:

- large power is required $(\approx 120 \mathrm{~W}$ each) to ensure proper functioning of two airborne transmitters;

- two transmitters require the use of two transmitting antennas, which, in turn, leads to additional requirements for electromagnetic compatibility and the optimal placement of these antennas on the surface of the spacecraft in order to reduce the influence of the spacecraft body on the energy parameters of the antennas;

- limited number of ground data receiving terminals that are capable of picking up a signal with a given energy;

- large-sized transmitting antennas.

\section{Antenna arrays for radio data communication of target information}

Data transmission to the Earth is carried out by the intravehicular information system (ISI) in four frequency bands. Each of these bands is covered by a separate antenna-feeder system, which uses low-profile low-cost low-directional antennas, for example, flat printed (or planar) antennas on a ceramic base, recursive and printed doublet antennas. According to the requirements applicable to the radio data communication, it is advisable to use $(8025 \ldots 8400 \mathrm{MHz}$ or $25500 \ldots 27000 \mathrm{MHz})$. However, the use of higher frequencies $(25500 \ldots 27000 \mathrm{MHz})$ leads to a number of difficulties:

- expensive measuring equipment;

- the worst energy parameters of the radio communication channel;

- a narrow antenna pattern requires accurate alignment and orientation of the spacecraft itself;

- increased cost of on-board equipment and antennas.

Thus, using the frequency range of $8025 \ldots 8400 \mathrm{MHz}$ is required.

Increasing requirements for data capacity, speed, weight and size parameters and energy characteristics of data communication radio systems lead to the need to develop wide frequency band antennas and provide the required directivity patterns. One of the most effective ways to increase the speed of data exchange is to find ways to build an on-board scanning antenna system. As an example of the practical application of such an antenna system, we can consider an antenna array of waveguide emitters built on the electroforming technology Fig. 1.

Fig. 2 shows the dependence of the standing wave ratio (SWR) on the frequency. The most important characteristics of antennas with circulating polarization are the polarization characteristics, which include the ellipticity coefficient and its dependence on spatial coordinates. Fig. 3 shows the dependence of the ellipticity coefficient on the angular coordinates, constructed for different frequencies of the operating range: 1 - $f=8 \mathrm{GHz}, 2-\mathrm{f}$ $=8.5 \mathrm{GHz}, 3-\mathrm{f}=9 \mathrm{GHz}$. 


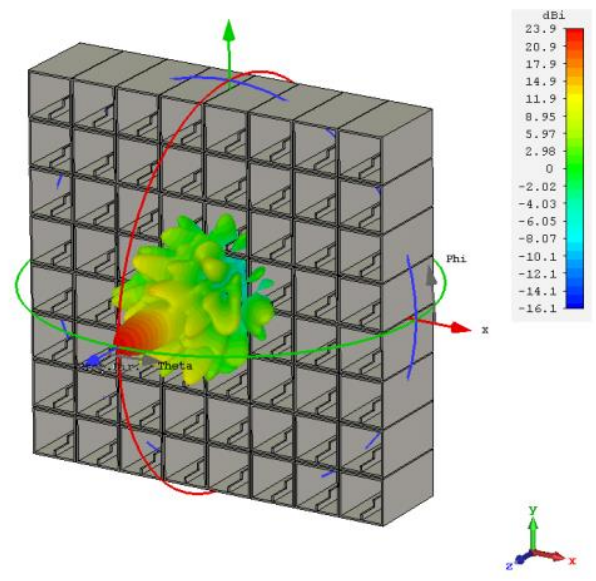

Fig. 1. Antenna array of circularly polarized waveguide emitters.

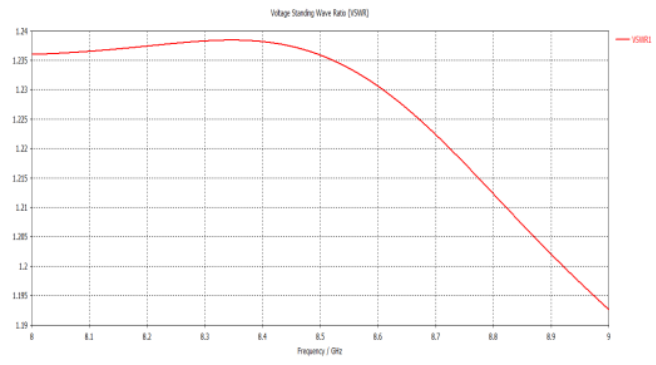

Fig. 2. Dependence of SWR on frequency.

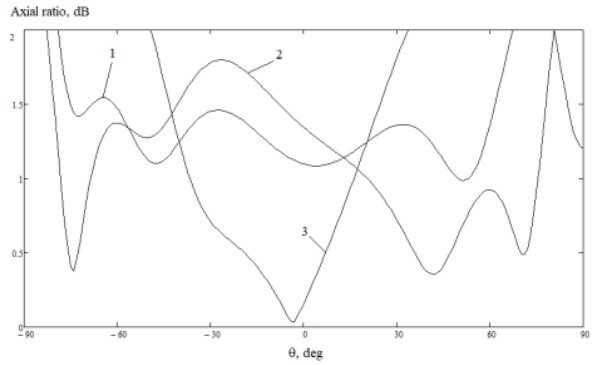

Fig. 3. Dependencies of the ellipticity coefficient on angular coordinates.

Along with waveguide and horn-type radiators with circular polarization, turnstile dipole emitters are widely used in satellite communication systems. Fig. 4 shows turnstile emitter model.

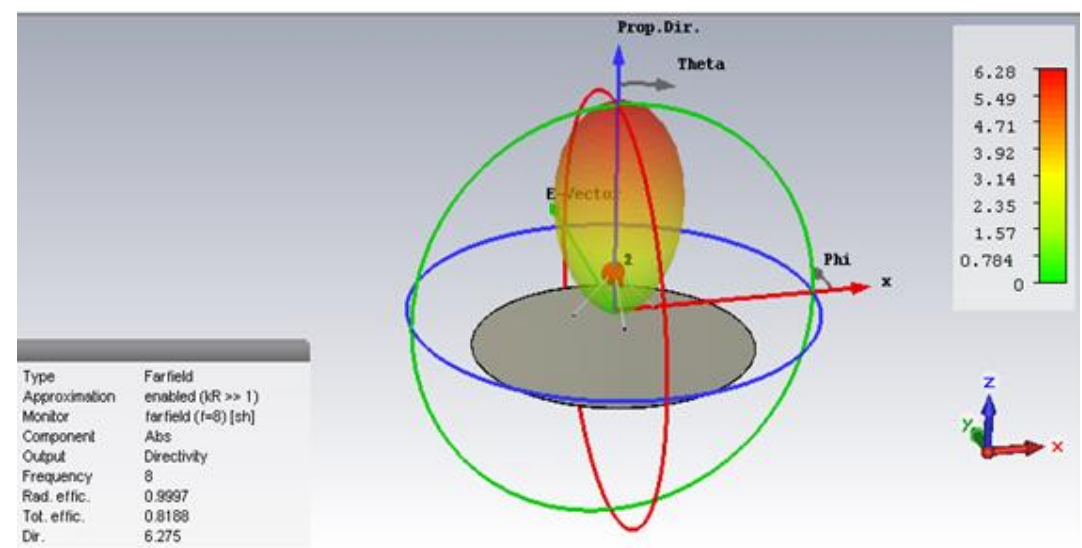

Fig. 4. Turnstile emitter model.

Fig. 5 shows a model of a circular concentric antenna array. We know the advantages of these arrays from reference books [6-8]. 


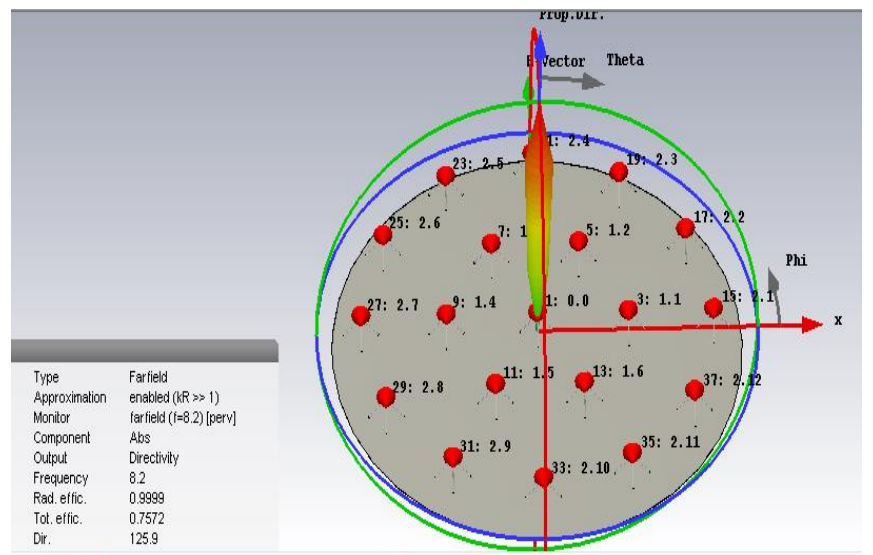

Fig. 5. Model of a circular concentric antenna array made of turnstile emitters.

Fig. 6 shows the dependencies of the ellipticity coefficient on the angular coordinates, constructed for different frequencies of the operating range: $\mathrm{a}-\mathrm{f}=8 \mathrm{GHz}, \mathrm{b}-\mathrm{f}=8.4 \mathrm{GHz}$.

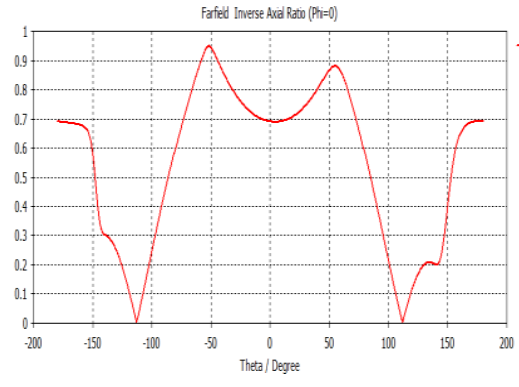

a)

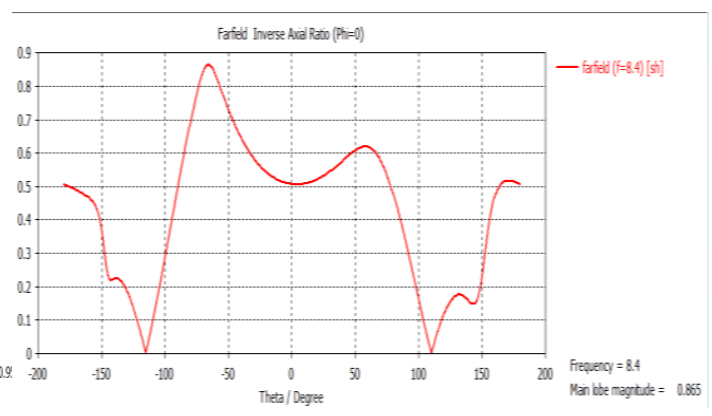

b)

Fig. 6. Dependencies of the ellipticity coefficient on angular coordinates.

Fig. 7 shows the space pattern factor with a beam deflection of $30^{\circ}$, Fig. 8 shows the dependence of the ellipticity coefficient on the angular coordinates at a frequency $f=8.2$ $\mathrm{GHz}$.

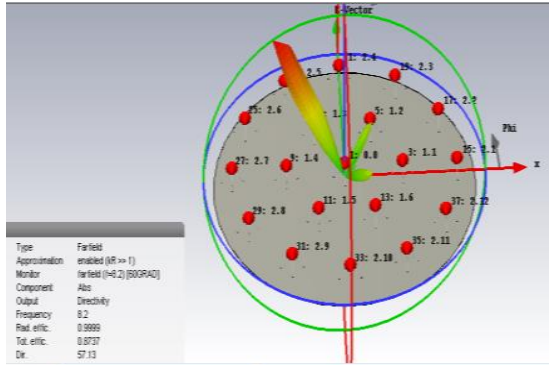

Fig. 7. Space pattern factor with a beam deflection of $30^{\circ}$.

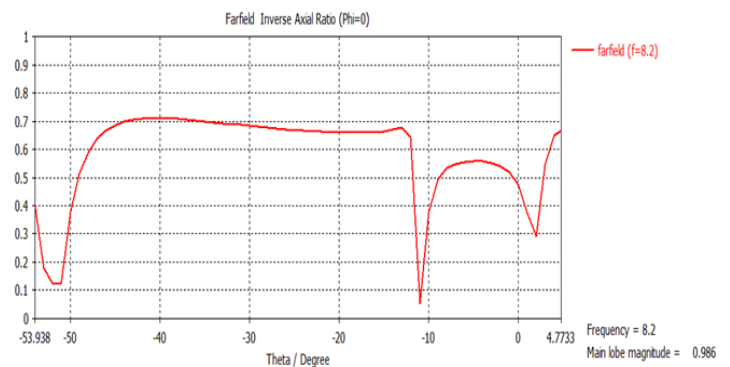

Fig. 8. Dependence of the ellipticity coefficient on the angular coordinates at a frequency $\mathrm{f}=8.2 \mathrm{GHz}$

A promising direction is the use of multilayer printing technology to create antenna systems for radio data communication of target information. 


\section{Conclusion}

Models of antenna curtains consisting of waveguide and turnstile emitters with circular polarization have been developed. The parametric optimization of emitters was carried out in order to improve the frequency characteristics of matching and directivity. The directivity characteristics and polarization characteristics of antenna arrays are determined.

\section{References}

1. B.A. Prigoda, V.S. Kokun'ko Aircraft antennas. M: Voenizdat, 120 p. (1964)

2. A.G. Generalov, E.V. Gadzhiyev. State and development prospects of airborne antenna-feeder devices for radio data communication of target information. Radiotekhnicheskiye i telekommunikatsionnyye sistemy. No. 2(30), pp. 44-52 (2018)

3. O.V. Bekrenev, A.K. Goncharov, S.I. Martynov. Antenna systems of receiving complexes for equipping UGDIS receiving stations. In book: Iosif'yanovskiye chteniya - 2017. Conference proceedings. October 26, pp. 240-241 (2017)

4. The space complex of operational monitoring of technological and natural emergencies "Kanopus-V" with the spacecraft "Kanopus-V" No. 1. M.: FGUP "NPP VNIIEM", 110 pp. (2011)

5. A.V. Vladimirov, R.S. Salikhov, N.A. Senik, S.A. Zolotoy. The space system for the operational monitoring of industrial and natural emergencies based on the Kanopus-V spacecraft and the Belarusian spacecraft // Electromechanical matters. VNIIEM studies. T. 105, pp. 49-57 (2008)

6. D.I. Voskresenskii, E.V. Ovchinnikova, Proc. of the XXVIII Moscow International Conference on Antenna theory and technology Russia, Moscow, September, (1998)

7. D.I. Voskresenskii, E.V. Ovchinnikova. Broadband antennas with wide angle undistorted scanning. - Antennas, No. 1 (42), (1999)

8. E.V. Ovchinnikova. Active phased array with wide-angle scanning. The 18th International Conference studies "Microwave Equipment and Telecommunication Technologies", Sevastopol, September 8-12 (2008) 